\title{
Acute spinal cord ischemia secondary to hypovolemic shock related to acute myocardial Infarction
}

\author{
Monique Boukobza* and Jean-Pierre Laissy \\ Department of Radiology, Assistance Publique -Hôpitaux de Paris, Bichat Hospital, 75018, Paris, France
}

\begin{abstract}
Acute spinal cord infarction is a rare condition accounting for $1 \%$ of stroke and should be recognized early. Especially in the presence of hypovolemic shock acute spinal cord infarction is easily misdiagnosed owing to its initial non-specific manifestation.

We report a case of a 40-year-old woman with a history of Type 1 diabetes mellitus, grade 2 hypertension and dyslipidemia, who presented 2 days after laparoscopic subtotal colonic resection for colonic polyposis a shock, related to myocardial infarct. Two days later a flaccid paraplegia was diagnosed. Magnetic resonance imaging findings were consistent with acute anterior spinal cord ischemia.
\end{abstract}

The clinical pattern, causes and Magnetic resonance imaging features of acute spinal cord infarction are discussed.

Patients with vascular risk factors should be carefully managed to minimise hypotensive episodes

\section{Introduction}

Acute spinal cord infarction (SCI) is a rare condition, accounting for $1 \%$ of stroke [1] and represents a diagnostic challenge. Most of them concern the territory of the anterior spinal artery (ASA) resulting in the so-called " anterior cord syndrome " or " ASA syndrome " or « centromedullar syndrome».

Since the clinical onset is non-specific, it may be misdiagnosed. SCI can lead to paralysis or paraparesis and carries a high rate of mortality: recognizing SCI early is crucial so that the causes producing ischemia can be corrected to limit infarction.

Acute SCI is caused by various conditions, the main cause being aortic procedures with a prevalence rate ranging 3\%-14\% [2].

However, SCI is idiopathic in $45 \%$ of cases $[1,3,4]$.

We describe a patient who developed permanent paraplegia following hypovolemic shock related to acute myocardial infarction.

\section{Case}

A 40-year-old woman, with a history of Type 1 diabetes mellitus, grade 2 hypertension and dyslipidemia, underwent laparoscopic subtotal colonic resection for colonic polyposis. The patient was hemodynamically stable in the immediate postoperative period.

On postoperative day 2 her level of consciousness decreased.

At arrival in an ICU : Glasgow Coma Score (GCS) of 14/15, a systolic blood pressure of $70 \mathrm{mmHg}$, heart rate, of $50 \mathrm{bpm}$, hypoxia ( $84 \%$ under $0^{2} 4 \mathrm{~L} / \mathrm{min}$ ). She was intubated, ventilated and sedated.

Electrocardiogram showed anterior wall myocardial infarction, and troponine US peaked at 109000 . Coronary angiography realized in emergency revealed occlusion of the right coronary artery which was promptly stented resulting in good distal flow. She was given aspirin, clopidogrel and heparin.
Two days later (postoperative day 4) she complained of being unable to move her legs and was found to be paraplegic (flaccid paraplegia and areflexia without pyramidal signs) with urinary retention, hypotonic anal sphincter and a T7 sensory level with preserved propioception and vibration sensation.

These findings were most consistent with acute SCI affecting the anterior spinal cord.

Body Angio-CT (ACT) showed no aortic dissection. Brain magnetic resonance imaging (MRI) showed no brain damage and post-contrast magnetic resonance angiography (MRA) of the cervical arteries no evidenced vertebral artery dissection.

Spinal MRI realized in emergency showed within the spinal cord at T2-T6 :T2 hyperintense anterior spinal lesion (Figures 1a-b), T1 hypointense without definite enhancement, and spinal cord swelling. Diffusion-weighted imaging shows a hypersignal at the same site (Figure 1c) with restriction diffusion on apparent coefficient diffusion (ADC) map (Figure 1d).

Blood cultures for gram-positive, gram-negative bacteria, and fungus were negative.

Serological markers of autoimmune disease (antinuclear antibody, antiextractable nuclear antigen antibody, antidouble stranded DNA antibody) and of thrombophilia (homocysteine levels, lupus anticoagulant, anticardiolipin immunoglobulin (Ig) G and IgM,

${ }^{\star}$ Correspondence to: Monique Boukobza, Department of Radiology, Assistance Publique -Hôpitaux de Paris, Bichat Hospital, 75018, Paris, France, Tel: + 33624 65 29 32; fax: + 33140258305 ; E-mail: m.boukobza@orange.fr

Key words: spinal cord ischemia, hypovolemic shock, myocardial infarction, MRI, anterior spinal syndrome

Received: August 06, 2018; Accepted: August 13, 2018; Published: August 20, 2018 


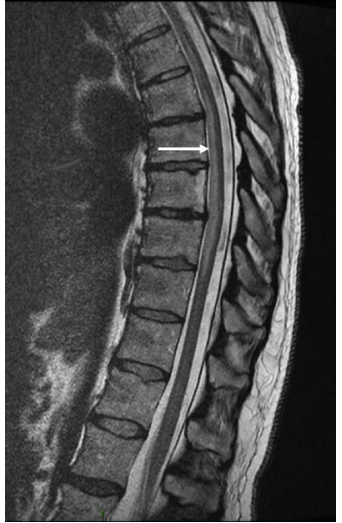

a

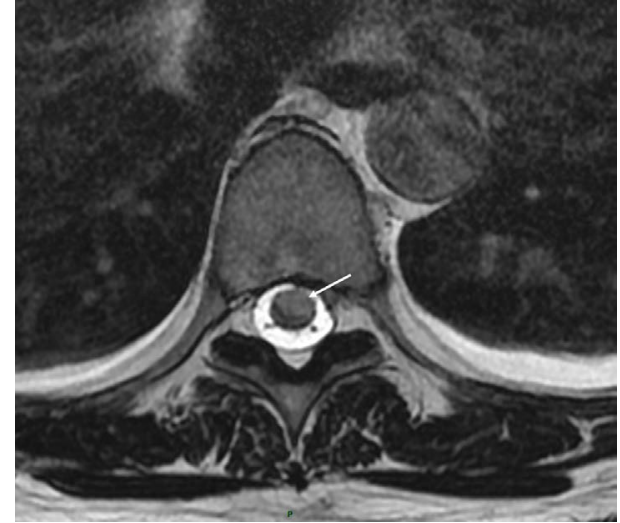

b

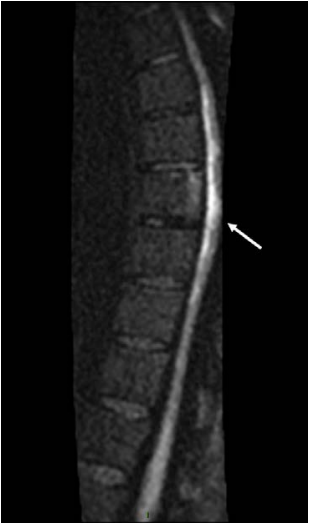

C

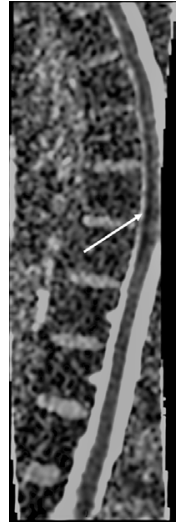

d

Figure 1.

1a. MRI (sagittal T2-weighted image) of the thoracic spine showing anterior hyperintensity in mid-thoracic cord (arrow).

1b. MRI (axial T2-weighted image) of the thoracic spine showing hyperintensity at the anterior 2/3 (arrow).

1c. MRI (Diffusion-weighted image, DWI) of the thoracic spine showing anterior hyperintensity in mid-thoracic cord (arrow).

1d. MRI (apparent coefficient diffusion (ADC) map) shows marked restriction diffusion at the site of the DWI hyperintensity, consistent with cytotoxic edema (arrow).

activated protein $\mathrm{C}$ resistance, protein $\mathrm{C}$, protein $\mathrm{S}$ and antithrombin III activity, factor II and V mutation, JAK 2 mutation) were negative. Serology markers including hepatitis B surface antigen, HIV, CMV, EBV, Lyme PCR were negative.

\section{TEP showed no abnormalities.}

On follow-up at six months after physical rehabilitation, there was no recuperation and the patient required intermittent urinary catheterization.

\section{Discussion}

Acute SCI can be caused by a variety of vascular events, including embolic disease, vasculitis, cardiac arrest, prolonged hypotension, e.g. due to cardiac failure or drugs, hypotension-induced surgery, atherosclerosis, cardiac embolism, aortic dissection, vertebral artery dissection, coagulopathy, trauma, cocaine abuse, epidural anesthesia, epidural infections, spondylodiscitis, spinal artery compression from herniated disks [5]. Complications after interventions to the aorta or its branches (aortic aneurysm repair) or spinal angiography represent $45 \%$ of SCI presentations [6,7].

In cases, several risk factors as hypertension, diabetes, atherosclerosis are associated. However, SCI is cryptogenic in $45 \%-50 \%$ of cases.

SCI affects predominantly the elderly, particularly in the presence of cardio-vascular risk factors.

SCI patients are younger and more often women compared to cerebral stroke patients $[8,9]$.

Most of SCI involve the anterior two-thirds of the cord which is the watershed supplied by the anterior spinal artery (ASA) and have distinct clinical pattern because of sparing of the dorsal columns.

The clinical pattern, named " anterior cord syndrome ", is usually marked by the acute onset of severe back pain ( $>80 \%$ of acute SCI are painful). SCI is characterized by paraplegia, areflexia, loss of pain, and temperature sensation, with preserved proprioception

In the current case, given the temporal relationship, the most obvious factor resonsable for anterior spinal cord ischemia was the hypotension secondary to anterior myocardial infarction (MI), even if the MI was successfully treated with resuscitation, stenting. This case shows also that a patient with MI can develop SCI without concomitant cerebral infarction.

Mild hypotension, hypotensive episodes, could also be harmful for a patient who is hypertensive for long.

A profound and sustained arterial hypotension may cause SCI, involving primarily the watershed area of the cord, between $\mathrm{T} 1$ and $\mathrm{T} 4$ cord segments [10].

Unfortunately, as our patient was sedated to assist ventilation, it was not possible to highlight the paralysis early enough. Patients with vascular risk factors should be carefully managed to minimise hypotensive episodes.

\section{Imaging}

MRI findings consist in a hyperintense signal on T2-weighted images with a "pencil-like" hyperintense lesion pattern on sagittal images. A "snake eye" or "owl's eye" appearance on axial images are typical of SCI. However, MRI aspect is often normal within 24 hours of onset. The features of SCI appear at day 2 of onset $[11,12]$.

Hyperintensity on DW with corresponding areas of hypointensity on ADC map (diffusion restriction), indicating cytotoxic edema can be valuable in the diagnosis of SCI (Figures 1c-d). The lesion on axial DWI has also a «snake eye » appearance. However absence of hyperintensity on DWI early in the disease, does not rule out the diagnosis $[13,14]$.

Post-contrast T1 sequence may be useful to differentiate SCI from inflammatory, infectious or tumoral lesions : SCI does not enhance after contrast at the acute stage.

$\mathrm{CT}$ and conventional angiography are not useful for the diagnosis.

\section{Differential diagnosis}

The differential diagnosis of acute spinal cord syndrome includes several nonischemic causes which can mimic spinal cord infarction.

Spinal cord compression caused by neoplasm or infectious lesions as spondyldiscitis and/or epidural abscess, epidural hematoma, have different MRI features. 
Myelitis and multiple sclerosis may present with similar MRI features, except for diffusion signal.

This case therefore most likely demonstrates SCI secondary to prolonged hypotension associated with anterior myocardial infarction.

This occurrence is rarely encountered in cardiovascular medicine.

\section{Treatment}

There are no clear guidelines for the treatment of spinal cord infarction [15]. It has recently suggested treatment with 250-500 mg acetylsalicylic acid (ASS) for SCI during the acute stage and a subsequent daily dose for secondary prevention is $100 \mathrm{mg}$ oral ASS. Treatment with rehabilitative therapy remains supportive.

\section{Prognosis}

Prognosis is based on the initial severity of deficits and on the extension of lesions on MRI examination.

\section{Conclusion}

Our case highlights an unusual complication of MI and emphasizes the importance of neurological examination following hypotensive episode in MI onset, to detect potentially modifiable causes of neurological injury.

Patients with severe atherosclerosis and vascular risk factors should be carefully managed to avoid hypotensive episodes.

Urgent MRI can be normal in the acute phase but is required to rule out spinal cord compression. Repeated MRI may confirm the diagnosis of SCI, if possible with application of DWI.

\section{References}

1. Novy J, Carruzzo A, Maeder P, Bogousslavsky J (2006) Spinal cord ischemia: clinical and imaging patterns, pathogenesis, and outcomes in 27 patients. Arch Neurol 63: 11131120. [Crossref]
2. Ullery BW, Cheung AT, Fairman RM, Jackson BM, Woo EY, et al. (2011) Risk factors, outcomes, and clinical manifestations of spinal cord ischemia following thoracic endovascular aortic repair. J Vasc Surg 54: 677-684. [Crossref]

3. Novy J (2012) Spinal cord syndromes. Front Neurol Neurosci 30: 195-198. [Crossref]

4. Weidauer S, Nichtweiß M, Hattingen E, Berkefeld J (2015) Spinal cord ischemia: aetiology, clinical syndromes and imaging features. Neuroradiology 57: 241-257. [Crossref]

5. Drummond JC, Lee RR, Owens EL (2012) Spinal cord ischemia occurring in association with induced hypotension for colonic surgery. Anesth Analg 114: 1297-1300. [Crossref]

6. Mawad ME, Rivera V, Crawford S, Ramirez A, Breitbach W (1990) Spinal cord ischemia after resection of thoracoabdominal aortic aneurysms: MR findings in 24 patients. AJNR Am J Neuroradiol 11:987-991. [Crossref]

7. Robertson CE, Brown RD Jr, Wijdicks EF, Rabinstein AA (2012) Recovery after spina cord infarcts: long-term outcome in 115 patients. Neurology 78: 114-121. [Crossref]

8. Romi F, Naess H (2016) Spinal Cord Infarction in Clinical Neurology: A Review of Characteristics and Long-Term Prognosis in Comparison to Cerebral Infarction. Eur Neurol 76: 95-98. [Crossref]

9. Romi F, Naess H (2016) Spinal Cord Infarction in Clinical Neurology: A Review of Characteristics and Long-Term Prognosis in Comparison to Cerebral Infarction. Eur Neurol 76: 95-98. [Crossref]

10. Diaz E, Morales H (2016) Spinal Cord Anatomy and Clinical Syndromes. Semin Ultrasound CT MR 37: 360-371. [Crossref]

11. Kumral E, Polat F, Gulluoglu H, Uzunköprü C, Tuncel R, et al. (2011) Spinal ischaemic stroke: clinical and radiological findings and short-term outcome. Eur J Neurol 18: 232-239. [Crossref]

12. Alves JN, Machado C, Taveira M, Soares-Fernandes J, Ferreira C et al. (2016) Teaching NeuroImages: Restricted diffusion "snake eyes appearance" in acute spinal cord ischemia. Neurology 86: e204-205. [Crossref]

13. Thurnher MM, Bammer R (2006) Diffusion-weighted MR imaging (DWI) in spinal cord ischemia. Neuroradiology 48: 795-801. [Crossref]

14. Artemis D, Wolf M, Blahak C, Szabo K, Hennerici MG, et al. (2017) Diagnostic and Prognostic Relevance of Magnetic Resonance Imaging and Electrophysiological Findings in Acute Spinal Ischemia. J Stroke Cerebrovasc Dis 26: 459-464. [Crossref]

15. Nardone R, Pikija S, Mutzenbach JS, Seidl M, Leis S, et al. (2016) Current and emerging treatment options for spinal cord ischemia. Drug Discov Today 21: 1632 1641. [Crossref]

Copyright: (C2018 Boukobza M. This is an open-access article distributed under the terms of the Creative Commons Attribution License, which permits unrestricted use, distribution, and reproduction in any medium, provided the original author and source are credited. 